\title{
APPROXIMATION BY NONFUNDAMENTAL SEQUENCES OF TRANSLATES
}

\author{
R. A. ZALIK
}

\begin{abstract}
For functions $f(t)$ satisfying certain growth conditions, we consider a sequence of the form $\left\{f\left(c_{n}-t\right)\right\}$, nonfundamental in $L_{2}(R)$, and find a representation for those functions which are in the closure of its linear span. Some theorems concerning degree of approximation are also proved.
\end{abstract}

In [1], we found necessary and sufficient conditions for a sequence of the form $\left\{f\left(c_{n}-t\right)\right\}$ to be fundamental in $L_{2}(R)$. In this paper, motivated by earlier research of L. Schwartz [2], and I. I. Hirschmann, Jr. [3] (see also J. Korevaar [4], W. A. J. Luxemburg and J. Korevaar [5, p. 35, Theorem 8.2], and Clarkson and Erdös [6]), we consider the nonfundamental case and find a representation of those functions which are in the $L_{2}(R)$ closure of the linear span of $\left\{f\left(c_{n}-t\right)\right\}$. Our result applies to a different class of functions than those considered by the above mentioned authors. The techniques developed to attack this problem are also applied to find a lower bound for the $L_{2}(R)$ distance from $f(c-t)$ to the linear span of $\left\{f\left(c_{r}-t\right) ; r=0, \ldots, n\right\}$, obtaining a result similar to $[5, \mathrm{p}$. 31, Theorem 7.1], [4, p. 363, Theorem 4], or [6, p. 6, Theorem 2]. Finally, we also prove a Jackson type theorem valid for a class of continuous functions defined on a bounded interval.

In what follows, $\left\{d_{n}\right\}$ will be a sequence of distinct real numbers, satisfying the following conditions:

$$
\left|c_{n}^{2}-c_{r}^{2}\right| \geqslant \rho|n-r| \quad(\rho>0) \text { and } \quad \sum^{\prime}\left|c_{n}\right|^{-2}<\infty .
$$

(By $\Sigma^{\prime}\left|c_{n}\right|^{-2}$ we denote the sum of all terms of the form indicated, with nonvanishing denominator.) Note that (1) is satisfied if, for instance

$$
\left|c_{n+1}\right| \geqslant \rho\left|c_{n}\right| \quad(\rho \geqslant \sqrt{2}) .
$$

Given a function $f(t)$, by $F(t)$ we shall denote its Fourier transform. Thus

$$
F(t)=(2 \pi)^{-1 / 2} \int_{R} \exp (x t i) f(t) d t .
$$

We shall assume that there are strictly positive numbers $\alpha, a$ and $b$, such that for $t$ real, $f(t)=O\left[\exp \left(-\alpha t^{2}\right)\right], F(t)=O\left[\exp \left(-a t^{2}\right)\right], t \rightarrow \infty$, and $\exp \left(-b t^{2}\right) / F(t)$ is in $L_{2}(R)$. By a theorem of Babenko, later generalized by Gel'fand and Šilov, we know that the growth condition on $f(t)$ can be replaced by the assumption that $F$ is an

Received by the editors January 19, 1979.

AMS (MOS) subject classifications (1970). Primary 30A62, 41A30, 42A64; Secondary 42 A68.

Key words and phrases. Approximation by sequences of translates, entire functions, Fourier inversion. 
entire function of order 2 and finite type (cf. [7, p. 238, Theorem 3]). Finally, if $f_{n}(t)=f\left(c_{n}-t\right)$, and $F_{n}(t)$ is its Fourier transform, it is readily seen that $F_{n}(-t)=$ $F(t) \exp \left(c_{n} t i\right)$; we shall denote by $S$ the linear span of the sequence $\left\{f_{n}\right\}$, and by $T$ the linear span of the sequence $\left\{F_{n}\right\}$.

Our first result is:

TheOREM 1. Assume $\left\{c_{n}\right\}$ satisfies (1). Then if the function $g(t)$ is in the $L_{2}(R)$ closure of $S$, it coincides a.e. on $R$ with a series of the form $\sum b_{n} f_{n}(t)$.

Theorem 1 is proved with the help of the following auxiliary proposition:

Lemma. Assume $\left\{c_{n}\right\}$ satisfies (1). Then there are continuous functions $p_{k}(t)=$ $p_{k}(t, \mu)$, having Fourier transforms $m_{k}(t)=m_{k}(t, \mu)$, satisfying the following conditions:

(a) Let $h(t)=\exp \left(-b t^{2}\right) /|F(t)|$; then for every $\mu<1 /(2 b)$ and positive,

$$
\left|m_{k}(t, \mu)\right| \leqslant d \exp \left[-(1 /(2 \mu)-b) t^{2}+\mu c_{k}^{2}\right] h(t),
$$

where $d$ is independent of $k$.

(b) $\int_{R} p_{k}(t) f_{n}(t) d x=\int_{R} m_{k}(t) F_{n}(t) d t=\delta_{k n}$ where $\delta_{k n}$ is Kronecker's delta.

(c) For $g(t)$ in $L_{2}(R)$, let $b_{k}(g)=\int_{R} p_{k}(t) g(t) d t$, then for any $\delta<\alpha$ and positive, there is a value of $\mu$ and a number $\gamma$ such that for all real $t$,

$$
\left|b_{n}(g) f_{n}(t)\right| \leqslant c^{2}\|g\|_{L_{2}(R)} \exp \left(-\delta c_{k}^{2}+\gamma t^{2}\right),
$$

where $c$ is independent of $k$, and if for this value of $\mu, S(g, t)=\sum b_{n}(g) f_{n}(t)$, then

$$
|S(g, t)| \leqslant M(t)\|g\|_{L_{2}(R)}, \quad \text { where } M(t)=c \exp \left(\gamma t^{2}\right) \sum \exp \left(-\delta c_{n}^{2}\right) .
$$

Using the preceding Lemma, we can prove:

TheOREM 2. Assume $\left\{c_{n}\right\}$ satisfies (1), and let $c$ be any real number not in the range of the sequence $\left\{c_{n}\right\}$. If $|c|=\left|c_{n}\right|$ for some $n$, let $m_{c}=1$; otherwise, let $m_{c}=\inf \mid 1-$ $\left(c / c_{n}\right)^{2} \mid$, the infimum being taken over the set of natural numbers. Let $d_{c}$ denote the $L_{2}(R)$ distance from $F(t) \exp (c t i)$ to $T$. Then there is a number $D>0$, independent of $c$ and $k$, such that $d_{c} \geqslant D m_{c}^{2} \exp \left(-c^{2} / 8 b\right)$.

REMARK. Since the Fourier transform is norm-preserving in $L_{2}(R), d_{c}$ also denotes the $L_{2}(R)$ distance from $f(c-t)$ to $S$. It should also be pointed out that the lower bound in Theorem 2 is not the best possible.

From Theorem 2 we obtain the following

COROLlaRY. If $c$ is not in the range of the sequence $\left\{c_{n}\right\}$, then neither $F(t) \exp (c t i)$ is in the $L_{2}(R)$ closure of $T$, nor is $f(c-t)$ in the $L_{2}(R)$ closure of $S$.

Finally, we have:

TheOREM 3. Assume that $\left\{c_{n}\right\}$ satisfies (1), and let $g(t)$ be a function in the $L_{2}(R)$ closure of $S$. Let $\left(a_{1}, b_{1}\right)$ be a bounded interval, assume $g(t)$ is continuous thereon, and let $d_{n}$ denote the uniform distance from $g(t)$ to the span of $\left\{f_{r}(t) ; r=0, \ldots, n\right\}$ in $\left(a_{1}, b_{1}\right)$. Then for any number $\delta, 0<\delta<\alpha$, there are numbers $D$ (independent of $n$ and $g$ ) and $\gamma$ (independent of $n)$, such that $d_{n} \leqslant D\|g\|_{L_{2}(R)} \exp \left(-\delta \rho^{n}\right)$. 
We shall use the following notation: $B y \Sigma^{(k)}$ and $\Pi^{(k)}$ we shall denote sums and products of the form indicated, $k$ th term deleted. For the theory of entire functions we shall refer to the book by R. P. Boas, Jr. [8].

Proof of Lemma. We shall only consider the case in which $c_{n} \neq 0$ for all $n$, the other case being similar. Let $r_{k}(z)=\Pi^{(k)}\left(1-z^{2} / c_{n}^{2}\right)$, and $\mu>0$. As in the proof of [5, p. 33, Lemma 7.2] (with $\left.\lambda_{n}=c_{n}^{2}\right)$, we see that the sequence $\left\{\exp \left[(\mu / 4) c_{k}^{2}\right] r_{k}\left(c_{k}\right)\right\}$ is bounded away from zero, say

$$
\exp \left[(\mu / 4) c_{k}^{2}\right]\left|r_{k}\left(c_{k}\right)\right| \geqslant D>0 .
$$

Clearly $r_{k}(z)=P_{k}(z) P_{k}(-z)$, where

$$
P_{k}(z)=\prod^{(k)} E\left(z / c_{n}, 1\right)=\prod^{(k)}\left(1-z / c_{n}\right) \exp \left(z / c_{n}\right) \text {. }
$$

If $n_{k}(r)$ denotes the number of elements in the sequence $\left\{c_{n}, n \neq k\right\}$ within the disk of radius $r$, and $n(r)$ is similarly defined for the whole sequence $\left\{c_{n}\right\}$, it is clear that $n_{k}(r) \leqslant n(r)$. In view of this inequality, setting $|z|=r$ and applying to $P_{k}(z)$ the same technique employed in the proof of [8, pp. 29-30, 2.10.13], we readily see there is a function $u(r)$ (the same for all $k$ ), such that $\lim _{r \rightarrow \infty} u(r)=0$, and

$$
\left|r_{k}(z)\right| \leqslant \exp \left[u(r) r^{2}\right]
$$

for all complex $z$. Setting

$$
q_{k}(z)=q_{k}(\mu, z)=(2 \pi)^{-1 / 2} \exp \left[(-\mu / 4)\left(z^{2}-c_{k}^{2}\right)\right] r_{k}(z) / r_{k}\left(c_{k}\right)
$$

we see that

$$
q_{k}\left(-c_{n}\right)=(2 \pi)^{-1 / 2} \delta_{k n}
$$

In view of (2) and (3), a straightforward computation shows that

$$
\begin{gathered}
\int_{R}\left|q_{k}(x+y i)\right|^{2} d x \leqslant d_{1}^{2} \exp \left[\mu\left(y^{2}+c_{k}^{2}\right)\right], \quad \text { and } \\
\int_{R}\left|(x+y i) q_{k}(x+y i)\right|^{2} d x \leqslant d_{2}^{2} \exp \left[\mu\left(y^{2}+c_{k}^{2}\right)\right],
\end{gathered}
$$

where $d_{1}$ and $d_{2}$ are independent of $k$ (they are, of course, dependent on $\mu$ ). Proceeding as in the proof of the necessity part of Theorem 3 in [1, pp. 304-305], we conclude that $q_{k}(z)$ is the Fourier transform of a function $h_{k}(t)=h_{k}(t, \mu)$ (i.e. $\left.q_{k}(z)=(2 \pi)^{-1 / 2} \int_{R} h_{k}(t) \exp (z t i) d t\right)$, such that $h_{k}(t)$ is continuous, and (for $t$ real),

$$
\left|h_{k}(t)\right| \leqslant d \exp \left[-t^{2} /(2 \mu)+\mu c_{k}^{2}\right]
$$

where $d$ is independent of $k$. Let $\mu<1 /(2 b)$. Then, if $m_{k}(t)=m_{k}(t, \mu)=$ $h_{k}(t) / F(t)$, and bearing in mind that $h(t)=\exp \left(-b t^{2}\right) / F(t)$ is in $L_{2}(R)$ by hypothesis, it is clear from (5) that

$$
\left|m_{k}(t)\right| \leqslant d \exp \left[-(1 /(2 \mu)-b) t^{2}+\mu c_{k}^{2}\right] h(t) .
$$

Let $p_{k}(t)$ be the inverse Fourier transform of $m_{k}(t)$. By Plancherel's formula and (6), we see that

$$
\int_{R}\left|p_{k}(t)\right|^{2} d t=\int_{R}\left|m_{k}(t)\right|^{2} d t \leqslant c^{2} \exp \left(2 \mu c_{k}^{2}\right)
$$

where $c$ is independent of $k$. 
From Plancherel's formula and (5), we also see that

$$
\begin{aligned}
\int_{R} p_{k}(t) f_{n}(t) d t & =\int_{R} p_{k}(t) f\left(c_{n}-t\right) d t=\int_{R} m_{k}(t) F(t) \exp \left(-c_{n} t i\right) d t \\
& =\int_{R} h_{k}(t) \exp \left(-c_{n} t i\right) d t=(2 \pi)^{1 / 2} q_{k}\left(-c_{n}\right)=\delta_{k n} .
\end{aligned}
$$

We have thus shown that

$$
b_{k}\left(f_{n}\right)=\delta_{k n}
$$

Let $g(t)$ be a function in $L_{2}(R)$. Applying the Cauchy-Schwartz inequality and (7), we see that

$$
\left|b_{n}(g) f\left(c_{n}-t\right)\right| \leqslant c\|g\|_{L_{2}(R)} \exp \left[\mu c_{n}^{2}-\alpha\left(c_{n}-t\right)^{2}\right] \text {. }
$$

Let $\delta$ be any number such that $0<\delta<\alpha$. Setting $\mu=\alpha-\delta-\epsilon$, where $0<\epsilon<\alpha$ $-\delta$, we see that $\mu c_{n}^{2}-\alpha\left(c_{n}-t\right)^{2}=-\delta c_{n}-\epsilon\left(c_{n}-t\right)^{2}+\gamma t^{2}$, whence we conclude that for this value of $\mu$,

$$
\left|b_{n}(g) f\left(c_{n}-t\right)\right| \leqslant c\|g\|_{L_{2}(R)} \exp \left(-\delta c_{n}^{2}+\gamma t^{2}\right),
$$

whence we readily conclude that

$$
|S(g, t)| \leqslant M(t)\|g\|_{L_{2}(R)},
$$

where $M(t)=c \exp \gamma t^{2} \Sigma \exp \left(-\delta c_{r}^{2}\right)$, and the conclusion follows from (6), (8), (9) and (10). Q.E.D.

Proof of Theorem 1. Assume that $g(t)$ is in the $L_{2}(R)$ closure of $S$. Let $\left\{g_{n}\right\}$ be a sequence of elements of $S$ that converges to $g(t)$ in the $L_{2}(R)$ distance. Taking if necessary a subsequence thereof, we can assume without loss of generality that $\left\{g_{n}\right\}$ converges to $g(t)$ a.e. in $R$.

From (8) we readily conclude that $S\left(g_{n}, t\right)=g_{n}(t)$. Applying (10), we thus see that

$$
\begin{aligned}
\left|g_{n}(t)-S(g, t)\right| & =\left|S\left(g_{n}, t\right)-S(g, t)\right| \\
& =\left|S\left(g_{n}-g, t\right)\right| \leqslant M(t)\left\|g_{n}-g\right\|_{L_{2}(R)} .
\end{aligned}
$$

Thus $S(g, t)=\lim _{n \rightarrow \infty} g_{n}(t)$, and therefore $g(t)=S(g, t)$, a.e., whence the conclusion follows. Q.E.D.

Proof of Theorem 2. Assume first that $|c| \neq\left|c_{n}\right|$ for all $n$. Since the sequence $\left\{c_{n}\right\}$ diverges, there is a number $k$ such that $\left|c_{k}\right|<|c|<\left|c_{k+1}\right|$. Let $d_{n}=c_{n}$ if $n<k, d_{k}=c$, and $d_{n}=c_{n+1}$ if $n>k$. Clearly (1) is also satisfied (with the same $\rho$ ) by the sequence $\left\{d_{n}\right\}$. Let $r(z)=\Pi\left(1-z^{2} / c_{n}^{2}\right)$, and $P(z)=\Pi^{(k)}\left(1-z^{2} / d_{n}^{2}\right)$. Clearly, $r(c)=\left(1-c^{2} / c_{k}^{2}\right)\left(1-c^{2} / c_{k+1}^{2}\right) P(c)$. Let $\mu>0$; inspection of the proof of $[5$, p. 33, Lemma 7.2] shows that

$$
\exp \left[(\mu / 4) c^{2}\right]|P(c)|=\exp \left[(\mu / 4) c^{2}\right]\left|P\left(d_{k}\right)\right|>D>0
$$

(where $D$ is independent of $c$ ), and therefore

$$
|r(c)|>m_{c}^{2} D .
$$


Let $q(z)=q(\mu, z)=\exp \left[-(\mu / 4) z^{2}\right] r(z)$, and $0<\mu<1 /(2 b)$. Proceeding again as in [1, pp. 304-305], we see that

$$
q(z)=(2 \pi)^{-1 / 2} \int_{R} m(t) F(t) \exp (z t i) d t,
$$

where $m(t)=m(\mu, t)$ is such that $|m(t)|<d \exp \left[-(1 /(2 \mu)-b) t^{2}\right] h(t)$, and $h(t)=$ $\exp \left(-b t^{2}\right) /|F(t)|$ is in $L_{2}(R)$; thus the $L_{2}(R)$ norm of $m(t)$ is independent of $c$. Since $q\left(-c_{n}\right)=0$, it readily follows from $[9$, p. 337, (V. 75)], that

$$
|q(c)|=\left|\int_{R} m(t) F(t) \exp (c t i) d t\right| \leqslant d_{c}\|m\|_{L_{2}(R)} .
$$

Since $\mu<1 /(2 b)$, and (12) implies that $|q(c)|>D m_{c}^{2} \exp \left[-(\mu / 4) c^{2}\right]$, the conclusion follows. If $|c|=\left|c_{k}\right|$ for some $k$, define $d_{n}=c_{n}$ if $n \neq k$, and $d_{k}=c\left(=-c_{k}\right)$. Thus if $r(z)$ is defined as above, $r(z)=(1-z / c) \Pi^{(k)}\left(1+z / d_{n}\right)$, and therefore $r(c)=2 \Pi^{(k)}\left(1+d_{k} / d_{n}\right)$. Since the sequence $\left\{d_{n}\right\}$ satisfies $(1)$, the conclusion follows as above. Q.E.D.

Proof of Theorem 3. Let $g(t)$ be a function in the $L_{2}(R)$ closure of $S$. From Theorem 1 we know that $g(t)=S(g, t)$ a.e. on $R$. However, it is readily seen from (9) and the continuity of the functions $f_{n}(t)$, that $S(g, t)$ is continuous on $R$, and therefore identical with $g(t)$ on $\left(a_{1}, b_{1}\right)$. Thus,

$$
g(t)=\sum_{r=0}^{\infty} b_{r}(g) f_{r}(t)
$$

thereon. From (9) and (1) we know that if $t$ is in $\left(a_{1}, b_{1}\right)$, and $\eta^{2}=\sup \left\{a_{1}^{2}, b_{1}^{2}\right\}$, then

$$
\begin{aligned}
\left|b_{r}(g) f_{r}(t)\right| & \leqslant c\|g\|_{L_{2}(R)} \exp \left(\gamma n^{2}\right) \exp \left(-\delta c_{r}^{2}\right) \\
& \leqslant c\|g\|_{L_{2}(R)} \exp \left(\gamma \eta^{2}+c_{0}^{2}\right) \exp \left(-\delta \rho^{r}\right) .
\end{aligned}
$$

Combining (13) and (14) we have

$$
\begin{aligned}
d_{n} & \leqslant\left|g(t)-\sum_{r=0}^{n} b_{r}(g) f_{r}(t)\right| \leqslant \sum_{r=n+1}^{\infty}\left|b_{r}(g) f_{r}(t)\right| \\
& \leqslant Q\|g\|_{L_{2}(R)} \sum_{r=n+1}^{\infty} \exp \left(-\delta \rho^{r}\right) \\
& =Q\|g\|_{L_{2}(R)}\left[\exp \left(-\delta \rho^{n+1}\right) /(1-\exp (-\delta \rho))\right],
\end{aligned}
$$

whence the conclusion follows. Q.E.D.

\section{REFERENCES}

1. R. A. Zalik, On approximation by shifts and a theorem of Wiener, Trans. Amer. Math. Soc. 243 (1978), 299-308.

2. L. Schwartz, Etude des sommes d'exponentielles réelles, 2nd ed., Hermann, Paris, 1959.

3. I. I. Hirschman, Jr., On approximation by non-dense sets of translates, Amer. J. Math. 73 (1951), 773-778.

4. J. Korevaar, $A$ characterization of the submanifold of $C[a, b]$ spanned by the sequence $\left\{x^{m *}\right\}$, Nederl. Akad. Wetensch. Proc. Ser. A 50 (1947), 750-758 = Indag. Math. 9 (1947), 360-368. 
5. W. A. J. Luxemburg and J. Korevaar, Entire functions and Müntz-Szász type approximation, Trans. Amer. Math. Soc. 157 (1971), 25-37.

6. J. A. Clarkson and P. Erdös, Approximation by polynomials, Duke Math. J. 10 (1943), 5-11.

7. I. M. Gel'fand and G. E. Silov, Fourier transforms of rapidly increasing functions and questions of the uniqueness of the solution of Cauchy's problem, Amer. Math. Soc. Transl. (2) 5 (1957), 221-274. (Transl. of Uspehi Mat. Nauk 8 (1953), 3-54.)

8. R. P. Boas, Jr., Entire functions, Academic Press, New York, 1954.

9. M. Cotlar and R. Cignoli, An introduction to functional analysis, North-Holland, Amsterdam; American Elsevier, New York, 1974.

Department of Mathematics, Auburn Universtty, Auburn, Alabama 36830 